\title{
Dimers on two-dimensional lattices
}

\author{
F. Y. Wu \\ Department of Physics \\ Mortheastern University \\ Boston, Massachusetts 02115, USA
}

October 22, 2018

\begin{abstract}
We consider close-packed dimers, or perfect matchings, on twodimensional regular lattices. We review known results and derive new expressions for the free energy, entropy, and the molecular freedom of dimers for a number of lattices including the simple-quartic $\left(4^{4}\right)$, honeycomb $\left(6^{3}\right)$, triangular $\left(3^{6}\right)$, kagomé $(3 \cdot 6 \cdot 3 \cdot 6)$, 3-12 $\left(3 \cdot 12^{2}\right)$ and its dual $\left[3 \cdot 12^{2}\right]$, and $4-8\left(4 \cdot 8^{2}\right)$ and its dual Union Jack $\left[4 \cdot 8^{2}\right]$ Archimedean tilings. The occurrence and nature of phase transitions are also analyzed and discussed.
\end{abstract}

Key words: Close-packed dimers, two-dimensional lattices, exact results, phase transitions. 


\section{Introduction}

A central problem in statistical physics and combinatorial mathematics is the enumeration of close-packed dimers, often referred to as perfect matchings in mathematical literature, on lattices which mimics the adsorption of diatomic molecules on a surface [1. A folklore in lattice statistics states that close-packed dimers can always be enumerated for two-dimensional lattices. Indeed, the seminal works of Kasteleyn [2] and Fisher and Temperley [3, 4] on the simple-quartic lattice produced the first exact solution. However, a search of the literature indicates that very little else has been published. This paper is an attempt to clarify the situation. Here we review known results and derive new expressions for the free energy, entropy, and molecular freedom for various Archimedean two-dimensional lattices. We also discuss analytic properties of the free energy. As we shall see, the task is not straightforward as previously thought, and the enumeration for many Archimedean lattices still remain open.

We consider a regular two-dimensional array of $N$ (= even) lattice points which can be covered by $N / 2$ dimers in the large $N$ limit. Denote the dimer weights by $\left\{z_{i}\right\}$ and define the generating function

$$
Z\left(\left\{z_{i}\right\}\right)=\sum_{\text {dimer coverings }} \prod_{i} z_{i}^{n_{i}}
$$

where the summation is over all dimer coverings and $n_{i}$ is the number of dimers with weight $z_{i}$. For a large lattice $Z\left(\left\{z_{i}\right\}\right)$ is expected to grow exponentially in $N$. Our goal is to compute the "free energy" per dimer

$$
f\left(\left\{z_{i}\right\}\right)=\lim _{N \rightarrow \infty} \frac{2}{N} \ln Z\left(\left\{z_{i}\right\}\right) .
$$

Setting $z_{i}=1$, the numerical value

$$
S=f(\{1\})
$$

is the entropy of adsoptions of diatomic molecules and

$$
W=\lim _{N \rightarrow \infty}\left[Z_{N}(\{1\})\right]^{2 / N}=e^{S}
$$

is often known as the per-dimer molecular freedom. These are quantities of primary interest in mathematics, physics, and chemistry. ${ }^{1}$

\footnotetext{
${ }^{1}$ In some earlier papers relevant quantities are defined on a per lattice site basis with $W_{\text {per site }}=\sqrt{W}$, etc.
} 
The following two integration formulas are useful for our purposes:

$$
\frac{1}{2 \pi} \int_{0}^{2 \pi} d \theta \ln (2 A+2 B \cos \theta+2 C \sin \theta)=\ln \left[A+\sqrt{A^{2}-B^{2}-C^{2}}\right]
$$

for real $A, B, C$, and

$$
\frac{1}{2 \pi} \int_{0}^{2 \pi} d \theta \ln \left|A+B e^{i \theta}\right|=\ln \max \{|A|,|B|\}
$$

for real or complex $A$ and $B$.

We have also the following result which holds generally for any graph, planar or not:

Proposition: Let $G$ be a bipartite graph consisting of two sets of equal number of vertices $A$ and $B$, with vertices in $A$ connected only to vertices in $B$, and vice versa. Let $G^{*}$ be a graph generated from $G$ by adding edges connecting vertices within one set. Let $Z_{G}$ and $Z_{G^{*}}$ be the respective dimer generating functions. Then we have the identity

$$
Z_{G^{*}}=Z_{G}
$$

Namely, the addition of edges connecting vertices within one set of vertices in a bipartite graph does not alter the dimer generating function.

Proof: Let the inserted edges connect $A$ vertices. In any dimer configuration in $G^{*}$, every $B$ vertex must be covered by a dimer and this dimer must end at an $A$ vertex. Since the numbers of $A$ and $B$ sites are equal, these $A B$-dimers cover all vertices. Thus, the inserted edges do not enter the picture. Q.E.D.

In ensuing sections individual lattices are considered in separate sections. The Archimedean nomenclature [5] of tilings are also indicated.

\section{The simple-quartic lattice $\left(4^{4}\right)$}

For a simple-quartic lattice with uniform dimer weights $z_{1}$ and $z_{2}$ in the two (horizontal and vertical) directions, we have [2] - 4] (see also section 4 below)

$$
f_{\mathrm{SQ}}\left(z_{1}, z_{2}\right)=\frac{1}{8 \pi^{2}} \int_{0}^{2 \pi} d \theta \int_{0}^{2 \pi} d \phi \ln \left[2\left(z_{1}^{2}+z_{2}^{2}-z_{1}^{2} \cos (\theta+\phi)-z_{2}^{2} \cos (\theta-\phi)\right]\right.
$$


The free energy $f_{\mathrm{SQ}}\left(z_{1}, z_{2}\right)$ is regular in $z_{1}$ and $z_{2}$.

Setting $z_{1}=z_{2}=1$ and making use of the integration identity (5), we obtain

$$
\begin{aligned}
S_{\mathrm{SQ}} & =\frac{1}{8 \pi^{2}} \int_{0}^{2 \pi} d \theta \int_{0}^{2 \pi} d \phi \ln [4(1-\cos \theta \cos \phi)] \\
& =\frac{1}{\pi} \int_{0}^{\pi / 2} d \theta \ln [2(1+\sin \theta)] \\
& =\frac{4}{\pi} \int_{0}^{\pi / 4} d \theta \ln (2 \cos \theta) \\
& =\frac{2}{\pi} G
\end{aligned}
$$

where the last step follows from the identity (4.224.5) of [7] and

$$
G=1-3^{-2}+5^{-2}-7^{-2}+\cdots=0.915965594 \ldots \ldots
$$

is the Catalan constant. It follows that we have

$$
\begin{aligned}
S_{\mathrm{SQ}} & =0.583121808 \ldots \\
W_{\mathrm{SQ}} & =1.791622812 \ldots
\end{aligned}
$$

\section{The honeycomb lattice $\left(6^{3}\right)$}

Kasteleyn [6] has pointed out that phase transitions can occur in dimer systems with anisotropic weights and cited the honeycomb lattice as an example. The honeycomb dimer problem describes a modified KDP model whose statistical property was later analyzed in details [8, 9. Here is a summary of the findings.

Let $z_{1}, z_{2}, z_{3}$ be the dimer weights along the three edge directions of the honeycomb lattice. We have [8] (see also section 4 below)

$$
\begin{array}{r}
f_{\mathrm{HC}}\left(z_{1}, z_{2}, z_{3}\right)=\frac{1}{8 \pi^{2}} \int_{0}^{2 \pi} d \theta \int_{0}^{2 \pi} d \phi \ln \left[z_{1}^{2}+z_{2}^{2}+z_{3}^{2}+2 z_{1} z_{2} \cos \theta\right. \\
\left.+2 z_{2} z_{3} \cos \phi+2 z_{3} z_{1} \cos (\theta-\phi) .\right]
\end{array}
$$

When one of the dimer weight dominates so that $z_{1}, z_{2}, z_{3}$ do not form a triangle, the free energy (11) is frozen and one has

$$
f_{\mathrm{HC}}\left(z_{1}, z_{2}, z_{3}\right)=\ln z_{i}, \quad z_{i} \geq z_{j}+z_{k}, \quad i, j, k \text { distinct. }
$$


The second derivatives of the free energy in the $\left\{z_{1}, z_{2}, z_{3}\right\}$-space exhibit an inverse square-root singularity near the phase boundaries $z_{i}=z_{j}+z_{k}$. We shall refer to this singular behavior as the KDP-type transition.

Setting $z_{1}=z_{2}=z_{3}=1$ and carrying out the $\phi$-integration, we obtain

$$
\begin{aligned}
S_{\mathrm{HC}} & =\frac{1}{8 \pi^{2}} \int_{0}^{2 \pi} d \theta \int_{0}^{2 \pi} d \phi \ln [3+2 \cos \theta+2 \cos \phi+2 \cos (\theta-\phi)] \\
& =\frac{1}{4 \pi} \int_{-\pi}^{\pi} d \theta \ln \frac{1}{2}[3+2 \cos \theta+|1+2 \cos \theta|] \\
& =\frac{1}{4 \pi} \int_{-2 \pi / 3}^{2 \pi / 3} d \theta \ln (2+2 \cos \theta) \\
& =\frac{2}{\pi} \int_{0}^{\pi / 3} d \theta \ln (2 \cos \theta) \\
& =0.323065947 \ldots \\
W_{\mathrm{HC}} & =1.381356444 \ldots
\end{aligned}
$$

The resemblance of the last integral in (13) with that in (9) is striking. We also remark that the entropy $S_{H C}$ is the same as that of the ground state of an isotropic antiferromagnetic Ising model [10. The integral in (14) was first obtained and evaluated by Wannier in a study of the latter problem more than half century ago [11].

Finally, we point out that the honeycomb free energy can also be evaluated when there exists a dimer-dimer interaction [12]. Analyses of the phase diagram and the associated critical behavior make use of the method of the Bethe ansatz and are fairly involved. Readers are referred to [12] for details.

\section{The checkerboard square lattice}

The checkerboard lattice is a simple-quartic lattice with anisotropic dimer weights $z_{1}, z_{2}, z_{3}$ and $z_{4}$ as shown in Fig. 1. Again, the solution of this problem was certainly known to Kasteleyn [6] who cited that the model exhibits phase transitions. The solution has also been mentioned by Montroll [13, and studied recently in some detail by Cohn, Kenyon and Popp [14]. Here we provide a concise analysis using previously known results on vertex models. 
Orient lattice edges as shown for which it is known [15] that the Kasteleyn clockwise odd sign rule [2] can be realized by setting $z_{2} \rightarrow i z_{2}, z_{4} \rightarrow i z_{4}$ in the evaluation of the Pfaffian. This permits us to take unit cells of two lattice sites as shown in Fig. 1. Since the cells form a rectangular array, following the standard procedure [13] one obtains its free energy given by the generally valid expression

$$
f\left(\left\{z_{i}\right\}\right)=\frac{1}{8 \pi^{2}} \int_{0}^{2 \pi} d \theta \int_{0}^{2 \pi} d \phi \ln \operatorname{det} F(\theta, \phi),
$$

where

$$
\begin{aligned}
F(\theta, \phi)= & M_{(0,0)}+M_{(1,0)} e^{i \theta}+a(-1,0) e^{-i \theta}+M_{(0,1)} e^{i \phi} \\
& +M_{(0,-1)} e^{-i \phi}+M_{(1,1)} e^{i(\theta+\phi)}+M_{(-1,-1)} e^{-i(\theta+\phi)}
\end{aligned}
$$

and the M's are matrices reflecting the orientation and connectivity of the edges.

For the checkerboard lattice we have ${ }^{2}$

$$
\begin{aligned}
& M_{(0,0)}=\left(\begin{array}{cc}
0 & z_{1} \\
-z_{1} & 0
\end{array}\right), \quad M_{(1,0)}=\left(\begin{array}{cc}
0 & 0 \\
-i z_{4} & 0
\end{array}\right), \quad M_{(-1,0)}=\left(\begin{array}{cc}
0 & i z_{4} \\
0 & 0
\end{array}\right) \\
& M_{(0,1)}=\left(\begin{array}{cc}
0 & 0 \\
i z_{2} & 0
\end{array}\right), \quad M_{(0,-1)}=\left(\begin{array}{cc}
0 & -i z_{2} \\
0 & 0
\end{array}\right) \\
& M_{(1,1)}=\left(\begin{array}{cc}
0 & 0 \\
z_{3} & 0
\end{array}\right), \quad M_{(-1,-1)}=\left(\begin{array}{cc}
0 & -z_{3} \\
0 & 0
\end{array}\right)
\end{aligned}
$$

Explicitly, this leads to

$$
\begin{gathered}
f_{\mathrm{CKB}}\left(z_{1}, z_{2}, z_{3}, z_{4}\right)=\frac{1}{8 \pi^{2}} \int_{0}^{2 \pi} d \theta \int_{0}^{2 \pi} d \phi \ln \left|z_{1}-i z_{4} e^{i \theta}+i z_{2} e^{i \phi}-z_{3} e^{i(\theta+\phi)}\right|^{2} \\
=\frac{1}{8 \pi^{2}} \int_{0}^{2 \pi} d \theta \int_{0}^{2 \pi} d \phi \ln \left[z_{1}^{2}+z_{2}^{2}+z_{3}^{2}+z_{4}^{2}+2\left(z_{1} z_{4}-z_{2} z_{3}\right) \sin \theta\right. \\
\left.\quad-2\left(z_{1} z_{2}-z_{3} z_{4}\right) \sin \phi-2 z_{1} z_{3} \cos (\theta+\phi)-2 z_{2} z_{4} \cos (\theta-\phi)\right]
\end{gathered}
$$

For $z_{1}=z_{3}, z_{2}=z_{4}$ the solution reduces to (8) for the simple-quartic lattice with uniform weights, and for $z_{4}=0$ the solution reduces to (11) for the

\footnotetext{
${ }^{2}$ The convention used here in writing down (17) is such that the direction to the right in Fig. 1 is the $(1,1)$-direction.
} 
honeycomb lattice. Comparing the second line of (18)) (after changing $\theta \rightarrow$ $\pi / 2-\theta, \phi \rightarrow \pi / 2-\phi$ ) with Eq. (16) of [16], we see that the checkerboard dimer model is completely equivalent to a free-fermion 6 -vertex model with weights

$$
\begin{array}{ll}
\omega_{1}=z_{4}, \quad \omega_{2}=z_{2}, & \omega_{3}=z_{1}, \quad \omega_{4}=z_{3} \\
\omega_{5} \omega_{6}=0, & \omega_{7} \omega_{8}=z_{1} z_{3}+z_{2} z_{4} .
\end{array}
$$

To analyze the free energy it is most convenient to apply the integration formula (6) to the first line of (18). This gives

$$
f_{\mathrm{CKB}}\left(z_{1}, z_{2}, z_{3}, z_{4}\right)=\frac{1}{2 \pi} \int_{0}^{2 \pi} d \theta \ln \max \left\{\left|z_{1}+i z_{4} e^{-i \theta}\right|,\left|i z_{2}+z_{3} e^{-i \theta}\right|\right\} .
$$

It is then seen that the free energy $f_{\mathrm{CKB}}$ is analytic in $z_{i}$, except when one of the weights dominates so that $z_{1}, z_{2}, z_{3}, z_{4}$ do not form a quadrilateral, namely,

$$
2 z_{i} \geq z_{1}+z_{2}+z_{3}+z_{4}, \quad i=1,2,3,4 .
$$

When this happens the free energy is frozen and

$$
f_{\mathrm{CKB}}\left(z_{1}, z_{2}, z_{3}, z_{4}\right)=\ln z_{i} .
$$

The free-fermion 6-vertex model with free energy (20) has been studied in details by $\mathrm{Wu}$ and Lin [17. ${ }^{3}$ It is found that, provided that either $z_{1} \neq z_{3}$ or $z_{2} \neq z_{4}$ (or both), namely, it is not the uniform simple-quartic model discussed in section 2 , the second derivatives of $f_{\mathrm{CKB}}$ in the $\left\{z_{1}, z_{2}, z_{3}, z_{4}\right\}$ space exhibit an inverse square-root singularity of the KDP-type transition near the phase boundary (22). This shows that the uniform model of section 2 is a unique degenerate case for which the free energy is analytic.

\section{The triangular lattice $\left(3^{6}\right)$}

The study of dimers on the triangular lattice has been of interest for many years (see, for example, [18 - 21]). Although an edge orientation of the

\footnotetext{
${ }^{3}$ The model (19) is transformed into one discussed in [17] after reversing all horizontal arrows in the vertex configurations. As found in [17, results reported therein apply to uniform as well as staggered models.
} 
triangular lattice satisfying the Kasteleyn clockwise-odd sign rule [2] for a Pfaffian evaluation has been given by Montroll [13], it seems that the closedform expression of the solution appeared in print only very recently [20, 21. Here, we provide a derivation of the solution using the Montroll edge orientation and analyze its physical properties.

Divide the lattice into unit cells containing two sites as shown in Fig. 2. Then, the free energy is given by the general expression (15) with

$$
\begin{array}{ll}
M_{(0,0)}=\left(\begin{array}{cc}
0 & z_{1} \\
-z_{1} & 0
\end{array}\right), \quad M_{(1,0)}=\left(\begin{array}{cc}
0 & 0 \\
z_{1} & 0
\end{array}\right), \quad M_{(-1,0)}=\left(\begin{array}{cc}
0 & -z_{1} \\
0 & 0
\end{array}\right), \\
M_{(0,1)}=\left(\begin{array}{cc}
z_{2} & -z_{3} \\
0 & -z_{2}
\end{array}\right), & M_{(0,-1)}=\left(\begin{array}{cc}
-z_{2} & 0 \\
z_{3} & z_{2}
\end{array}\right), \\
M_{(1,1)}=\left(\begin{array}{cc}
0 & 0 \\
z_{3} & 0
\end{array}\right), & M_{(-1,-1)}=\left(\begin{array}{cc}
0 & -z_{3} \\
0 & 0
\end{array}\right) .
\end{array}
$$

This leads to

$$
\begin{aligned}
& f_{\mathrm{TRI}}\left(z_{1}, z_{2}, z_{3}\right) \\
= & \frac{1}{8 \pi^{2}} \int_{0}^{2 \pi} d \theta \int_{0}^{2 \pi} d \phi \ln 2\left[z_{1}^{2}+z_{2}^{2}+z_{3}^{2}-z_{1}^{2} \cos \theta-z_{2}^{2} \cos 2 \phi+z_{3}^{2} \cos (\theta+2 \phi)\right] \\
= & \frac{1}{8 \pi^{2}} \int_{0}^{2 \pi} d \alpha \int_{0}^{2 \pi} d \beta \ln 2\left[z_{1}^{2}+z_{2}^{2}+z_{3}^{2}+z_{1}^{2} \cos \alpha+z_{2}^{2} \cos \beta+z_{3}^{2} \cos (\alpha+\beta)\right],
\end{aligned}
$$

where in the last step we have changed variables to $\alpha=\pi-\theta, \beta=\pi-2 \phi$. The free energy (24) is of the form of that of a free-fermion 8-vertex model considered by Fan and Wu [16]. Comparing (24) with Eq. (16) of [16], one obtains the free-fermion vertex weights $\omega_{1}, \cdots, \omega_{8}$ as given by

$$
\begin{aligned}
\omega_{1}^{2}+\omega_{2}^{2}+\omega_{3}^{2}+\omega_{4}^{2} & =2\left(z_{1}^{2}+z_{2}^{2}+z_{3}^{2}\right) \\
\omega_{1} \omega_{3}-\omega_{2} \omega_{4} & =z_{1}^{2} \\
\omega_{1} \omega_{4}-\omega_{2} \omega_{3} & =z_{2}^{2} \\
\omega_{3} \omega_{4}-\omega_{1} \omega_{2} & =z_{3}^{2} \\
\omega_{5} \omega_{6} & =\omega_{1} \omega_{2} \\
\omega_{7} \omega_{8} & =\omega_{3} \omega_{4} .
\end{aligned}
$$

It was found [16] that the model exhibits a transition at the critical point $\left(-\omega_{1}+\omega_{2}+\omega_{3}+\omega_{4}\right)\left(\omega_{1}-\omega_{2}+\omega_{3}+\omega_{4}\right)\left(\omega_{1}+\omega_{2}-\omega_{3}+\omega_{4}\right)\left(\omega_{1}+\omega_{2}+\omega_{3}-\omega_{4}\right)=0$, 
and that the transition is of a KDP-type transition with an inverse squareroot singularity in the second derivative of the free energy if $\omega_{1} \omega_{2} \omega_{3} \omega_{4}=0$, and an Ising-type transition with a logarithmic singularity in the second derivative if $\omega_{1} \omega_{2} \omega_{3} \omega_{4} \neq 0$.

We can solve for the $\omega$ 's by forming linear combinations of the equalities in (25) to obtain

$$
\begin{aligned}
\left(-\omega_{1}+\omega_{2}+\omega_{3}+\omega_{4}\right)^{2} & =4 z_{3}^{2} \\
\left(\omega_{1}-\omega_{2}+\omega_{3}+\omega_{4}\right)^{2} & =4\left(z_{1}^{2}+z_{2}^{2}+z_{3}^{2}\right) \\
\left(\omega_{1}+\omega_{2}-\omega_{3}+\omega_{4}\right)^{2} & =4 z_{2}^{2} \\
\left(\omega_{1}+\omega_{2}+\omega_{3}-\omega_{4}\right)^{2} & =4 z_{1}^{2} .
\end{aligned}
$$

This leads to the explicit solution

$$
\begin{aligned}
& \omega_{1}=\frac{1}{2}\left[z_{1}+z_{2}-z_{3}+\sqrt{z_{1}^{2}+z_{2}^{2}+z_{3}^{2}}\right] \\
& \omega_{2}=\frac{1}{2}\left[z_{1}+z_{2}+z_{3}-\sqrt{z_{1}^{2}+z_{2}^{2}+z_{3}^{2}}\right] \\
& \omega_{3}=\frac{1}{2}\left[z_{1}-z_{2}+z_{3}+\sqrt{z_{1}^{2}+z_{2}^{2}+z_{3}^{2}}\right] \\
& \omega_{4}=\frac{1}{2}\left[-z_{1}+z_{2}+z_{3}+\sqrt{z_{1}^{2}+z_{2}^{2}+z_{3}^{2}}\right]
\end{aligned}
$$

using which one verifies that we have $\omega_{1} \omega_{2} \omega_{3} \omega_{4} \neq 0$. This implies that the triangular dimer model exhibits Ising-type transitions at

$$
z_{i}=0, \quad i=1,2,3 .
$$

Namely, the uniform simple-quartic model discussed in section 2 is the critical manifold of the triangular model.

Setting $z_{1}=z_{2}=z_{3}=1$, we obtain

$$
\begin{aligned}
S_{\mathrm{TRI}} & =\frac{1}{8 \pi^{2}} \int_{0}^{2 \pi} d \alpha \int_{0}^{2 \pi} d \beta \ln [6+2 \cos \alpha+2 \cos \beta+2 \cos (\alpha+\beta)] \\
& =\frac{1}{2 \pi} \int_{0}^{\pi} d \alpha \ln \left[3+\cos \alpha+\sqrt{7+4 \cos \alpha+\cos ^{2} \alpha}\right] \\
& =0.857189074 \ldots \\
W_{\mathrm{TRI}} & =2.356527353 \ldots
\end{aligned}
$$


It is of interest to note that an integral similar to (30),

$$
\begin{aligned}
S_{\mathrm{SPT}} & =\frac{1}{4 \pi^{2}} \int_{0}^{2 \pi} d \alpha \int_{0}^{2 \pi} d \beta \ln [6-2 \cos \alpha-2 \cos \beta-2 \cos (\alpha+\beta)] \\
& =\frac{1}{\pi} \int_{0}^{\pi} d \alpha \ln \left[3-\cos \alpha+\sqrt{7-8 \cos \alpha+\cos ^{2} \alpha}\right] \\
& =1.615329736 \ldots,
\end{aligned}
$$

which gives the per-site free energy of spanning trees on the triangular lattice 22, 23, is reducible to a simple numerical series akin to the Catalan constant. The reduction involves the mapping of the spanning tree problem to a Potts model and in turn to an $F$ model on the triangular lattice [24]. After some steps [25] the spanning tree entropy (31) becomes

$$
S_{\mathrm{SPT}}=\frac{3 \sqrt{3}}{\pi}\left(1-5^{-2}+7^{-2}-11^{-2}+13^{-2}-\ldots\right)=1.615329736 \ldots
$$

This suggests the possibility that other entropy expressions can perhaps be similarly reduced.

\section{The kagomé lattice $(3 \cdot 6 \cdot 3 \cdot 6)$}

The kagomé lattice is shown in Fig. 3 with dimer weights $z_{1}, z_{2}, z_{3}$ along the 3 edge directions. The study of the molecular freedom for the kagomé lattice has been a subject matter of interest for many years (see, for example, [26, 27]), but most of the studies have been numerical or approximate.

The kagomé free energy has been computed recently 28] by using its equivalence to a staggered 8-vertex model solved by Hsue, et. al [29]. The solution is found to be surprisingly simple and is given by

$$
f_{\mathrm{KG}}\left(z_{1}, z_{2}, z_{3}\right)=\frac{1}{3} \ln \left(4 z_{1} z_{2} z_{3}\right) .
$$

The solution (33) differs fundamentally from those of other lattices as it does not have a series expansion. This explains why most of other approaches, which are invariably based on series expansions, are not very effective. ${ }^{4}$

\footnotetext{
${ }^{4}$ However, the series expansion scheme is recovered if one introduces further symmetrybreaking weights [28].
} 
From (33) we now have

$$
\begin{aligned}
S_{\mathrm{KG}} & =\frac{2}{3} \ln 2=0.462098120 \ldots \\
W_{\mathrm{KG}} & =2^{2 / 3}=1.587401051 \ldots
\end{aligned}
$$

\section{$7 \quad$ The 3 -12 lattice $\left(3 \cdot 12^{2}\right)$}

The 3-12 lattice is shown in Fig. 4. We consider the case of six different dimer weights $x, y, z, u, v, w$.

The 3-12 lattice has been used by Fisher [30] in a dimer formulation of the Ising model. Using Fisher's edge orientation which we show in Fig. 4, one finds the free energy given by the general expression (15) with

$$
F(\theta, \phi)=\left(\begin{array}{cccccc}
0 & x & z & 0 & -w e^{-i \theta} & 0 \\
-x & 0 & y & 0 & 0 & -v e^{-i \phi} \\
-z & -y & 0 & u & 0 & 0 \\
0 & 0 & -u & 0 & z & y \\
w e^{i \theta} & 0 & 0 & -z & 0 & x \\
0 & w e^{i \phi} & 0 & -y & -x & 0
\end{array}\right)
$$

Namely,

$$
\begin{aligned}
& f_{3-12}(x, y, z ; u, v, w) \\
&= \frac{1}{3} \cdot \frac{1}{8 \pi^{2}} \int_{0}^{2 \pi} d \theta \int_{0}^{2 \pi} d \phi \ln \left[\omega_{1}^{2}+\omega_{2}^{2}+\omega_{3}^{2}+\omega_{4}^{2}+2\left(\omega_{1} \omega_{3}-\omega_{2} \omega_{4}\right) \cos \theta\right. \\
&\left.\quad+2\left(\omega_{1} \omega_{4}-\omega_{2} \omega_{3}\right) \cos \phi+2\left(\omega_{3} \omega_{4}-\omega_{1} \omega_{2}\right) \cos (\theta-\phi)\right]
\end{aligned}
$$

where the factor $1 / 3$ is due to the fact that there are 3 dimers per unit cell, and

$$
\omega_{1}=x^{2} u, \quad \omega_{2}=u v w, \quad \omega_{3}=y^{2} w, \quad \omega_{4}=z^{2} v .
$$

The free energy (37) is again of the form of that of a free-fermion 8-vertex model with vertex weights $\omega_{1}, \omega_{2}, \omega_{3}, \omega_{4}, \omega_{5} \omega_{6}=\omega_{3} \omega_{4}$, and $\omega_{7} \omega_{8}=\omega_{1} \omega_{2}$ and the critical point (26). For the Ising model with interactions $K_{1}$ and $K_{2}$ [30], for example, we have $x=y=z=u=1, v=\tanh K_{1}, w=\tanh K_{2}$. It is then verified that the critical condition $\omega_{1}=\omega_{2}+\omega_{3}+\omega_{4}$ can be realized and gives the known Ising critical point

$$
\sinh 2 K_{1} \sinh 2 K_{2}=1 .
$$


Finally, setting $x=y=z=u=v=w=1$, we obtain

$$
\begin{aligned}
S_{3-12} & =\frac{1}{3} \ln 2=0.231049060 \ldots \\
W_{3-12} & =2^{1 / 3}=1.259921049 \ldots
\end{aligned}
$$

\section{The $4-8$ lattice $\left(4 \cdot 8^{2}\right)$}

Dimer models on the 4-8 lattice shown in Fig. 5 have been used to describe phase transitions in physical systems 31] - 33]. By setting $u=v$, for example, the dimer model describes the phase transition in the layered hydrogen-bonded $\mathrm{SnCl}^{2} \cdot 2 \mathrm{H}_{2} \mathrm{O}$ crystal [32].

Orient the lattice as shown, and this leads to the free energy (15) with

$$
F(\theta, \phi)=\left(\begin{array}{cccc}
0 & x e^{i \theta} & -v & -u \\
-x e^{-i \theta} & 0 & u & -v \\
v & -u & 0 & y e^{i \phi} \\
u & v & -y e^{-i \phi} & 0
\end{array}\right)
$$

Namely,

$$
\begin{aligned}
f_{4-8}(x, y ; u, v)=\frac{1}{2} & \cdot \frac{1}{8 \pi^{2}} \int_{0}^{2 \pi} d \theta \int_{0}^{2 \pi} d \phi \ln \left[x^{2} y^{2}+\left(u^{2}+v^{2}\right)^{2}\right. \\
& \left.-2 x y u^{2} \cos (\theta+\phi)-2 x y v^{2} \cos (\theta-\phi)\right] .
\end{aligned}
$$

The free energy (40) is also of the form of the that of a free-fermion 8-vertex model [16] and exhibits an Ising type transition at

$$
u^{2}+v^{2}=x y
$$

Setting $x=y=u=v=1$, one obtains

$$
\begin{aligned}
S_{4-8} & =\frac{1}{16 \pi^{2}} \int_{0}^{2 \pi} d \theta \int_{0}^{2 \pi} d \phi \ln [5-2 \cos (\theta+\phi)-2 \cos (\theta-\phi)] \\
& =\frac{1}{16 \pi^{2}} \int_{0}^{2 \pi} d \theta \int_{0}^{2 \pi} d \phi \ln [5-4 \cos \theta \cos \phi] \\
& =\frac{1}{2 \pi} \int_{0}^{\pi / 2} d \theta \ln \left[\frac{5+\sqrt{25-16 \cos ^{2} \theta}}{2}\right] \\
& =0.376995650 \ldots \\
W_{4-8} & =1.457897968 \ldots
\end{aligned}
$$




\section{The Union Jack lattice $\left[4 \cdot 8^{2}\right]$}

The Union Jack lattice shown in Fig. 6(a) is the dual of the 4-8 lattice. It is constructed by inserting diagonal edges with weights $u$ and $v$ to a checkerboard lattice. Since the checkerboard lattice is bipartite and the inserted diagonals connect vertices of one sublattice only, the Proposition established in section 1 now implies the identity

$$
Z_{\mathrm{UJ}}\left(z_{1}, z_{2}, z_{3}, z_{4} ; u, v\right)=Z_{\mathrm{CKB}}\left(z_{1}, z_{2}, z_{3}, z_{4}\right)
$$

That is, the solution for the Union Jack lattice is identical to that of a simple-quartic lattice as if the $u, v$ edges were absent. Particularly, they have identical entropy and molecular freedom.

\section{The $\left[3 \cdot 12^{2}\right]$ tiling lattice}

The $\left[3 \cdot 12^{2}\right]$ tiling lattice shown in Fig. $6(\mathrm{~b})$ is the dual of the 3-12 lattice. It consists of two sets of vertices, set $A$ vertices each of which having coordination number 3 and set $B$ vertices each having coordination number 12 . The number of $A$ vertices is twice that of $B$, and the $A$ vertices are connected to $B$ vertices only.

The lattice $\left[3 \cdot 12^{2}\right]$ does not admit dimer coverings. This follows from the fact that in a proper dimer covering each $A$ vertex must by covered by a dimer and the dimer must end at a $B$ vertex. This means some $B$ vertices will have more than one dimers and this is not possible. Thus, there is no proper dimer coverings and the generating function $Z_{\left[3 \cdot 12^{2}\right]}$ is identically zero.

\section{Summary and Acknowledgments}

We have presented analytic and numerical results on the free energy, entropy, and molecular freedom for close-packed dimers on two-dimensional lattices which have unit cells arranged on a rectangular array. For the anisotropic checkerboard lattice the free energy is found to exhibit a KDP-type singularity, except in the degenerate case of uniform dimer weights the free energy is analytic. For the triangular lattice the free energy is analytic for nonzero $z_{i}$ and is critical at $z_{i}=0$. For 4-8 and 3-12 lattices the dimer models exhibit Ising-type transitions. 


\begin{tabular}{|l|l|c|c|}
\hline Lattice & Entropy $S$ & $\begin{array}{c}\text { Molecular freedom } \\
W=e^{S}\end{array}$ & $\begin{array}{l}\text { Phase } \\
\text { transitions }\end{array}$ \\
\hline \hline Honeycomb $\left(6^{3}\right)$ & 0.323065947 & 1.381356444 & KDP \\
Checkerboard $\left(4^{4}\right)$ & 0.583121808 & 1.791622812 & KDP \\
Triangular $\left(3^{6}\right)$ & 0.857189074 & 2.356527353 & Ising \\
Kagomé $(3 \cdot 6 \cdot 3 \cdot 6)$ & 0.462098120 & 1.587401051 & None \\
$3-12\left(3 \cdot 12^{2}\right)$ & 0.231049060 & 1.259921049 & Ising \\
$4-8\left(4 \cdot 8^{2}\right)$ & 0.376995650 & 1.457897968 & Ising \\
Union Jack $\left[4 \cdot 8^{2}\right]$ & 0.583121808 & 1.791622812 & Ising \\
{$\left[3 \cdot 12^{2}\right]$} & no dimer coverings & no dimer coverings & None \\
\hline
\end{tabular}

Table 1: Summary of numerical results. Phase transitions occur for anisotropic dimer weights

Numerical results on the entropy and molecular freedom are summarized in Table 1. We observe that the entropy is not necessarily a monotonic function of the coordination number of the lattice. Analyses of other Archimedean tiling lattices still remains open.

I would like to thank R. Shrock for introducing me to Archimedean tilings and for comments and a critical reading of the manuscript, J. Propp for calling my attention to Ref. [14, and F. Hucht for pointing a numerical error in Eq. (32) in an earlier version of the manuscript. The assistance of W. T. Lu in preparing the figures is also gratefully acknowledged. Work has been supported in part by NSF Grant DMR-9980440. 


\section{References}

[1] R. H. Fowler and G. S. Rushbrooke, An attempt to extend the statistical theory to perfect solutions, Trans. Faraday Soc. 33, 1272-1294 (1937).

[2] P. W. Kasteleyn, The statistics of dimers on a lattice, Physica 27, 12091225 (1961).

[3] H. N. V. Temperley and M. E. Fisher, Dimer problem in statistical mechanics - An exact result, Phil. Mag. 6, 1061-1063 (1961).

[4] M. E. Fisher, Statistical mechanics of dimers on a plane lattice, Whys. Rev. 124, 1664-1672 (1961).

[5] See, for example, B. Garünbaum and G. C. Shephard, Tilings and Patterns (W. H. Freeman, New York 1987).

[6] P. W. Kasteleyn, Dimer statistics and phase transitions, J. Math. Phys. 4, 287-293 (1963).

[7] I. S. Gradshteyn and I. M. Ryzhik, Tables of Integrals, Series and Products (Academic Press, San Diego 2000).

[8] F. Y. Wu, Remarks on the modified potassium dihydrogen phosphate model of a ferroelectric, Phys. Rev. 168, 539-543 (1968).

[9] F. Y. Wu, Exactly soluble model of the ferroelectric phase transition in two dimensions, Phys. Rev. Lett. 18 605-607 (1967).

[10] See, for example, H. W. J. Blöte and H. J. Hilhorst, Roughening transitions and the zero-temperature triangular Ising antiferromagnet, J. Phys. A 15, L631-L637 (1982).

[11] G. H. Wannier, Antiferromagnetism. The triangular Ising net, Phys. Rev. 79, 357-364 (1950).

[12] H. Y. Huang, F. Y. Wu, H. Kunz, and D. Kim, Interacting dimers on the honeycomb lattice: An exact solution of the 5-vertex model, Physica A 228, 1-32 (1966).

[13] E. W. Montroll, Lattice Statistics, in Applied Combinatorial Mathematics, Ed. E. F. Beckenbach (John Wiley and Sons, New York 1964). 
[14] H. Cohn, R. Kenyon and J. Propp, A variational principle for domino tilings, J. AMS 14, 297-346 (2001).

[15] T. T. Wu, Dimers on rectangular lattices, J. Math. Phys. 3, 1265-1266 (1962).

[16] C. Fan and F. Y. Wu, General lattice model of phase transitions, Phys. Rev. B 2, 723-733 (1970).

[17] F. Y. Wu and K. Y. Lin, Staggered ice-rule vertex model - The Pfaffian solution, Phys. Rev. B 12, 419-428 (1975).

[18] J. F. Nagle, New series-expansion method for the dimer problem, Phys. Rev. 152, 190-197 (1966).

[19] A. J. Phares and F. J. Wunderlich, Thermodynamics and molecular freedom of dimers on plane triangular lattices, J. Math. Phys. 27, 10991109 (1985).

[20] R. Kenyon, The planar dimer model with boundary: a survey, Directions in Mathematical Quasicrystals, CRM Monogr. Ser. Amer. Math. Soc. 13, 307-328 (2000).

[21] P. Fendley, R. Moessner, and S. L. Sondhi, Classical dimer model on the triangular lattice, ArXive: cond-mat/0206159.

[22] F. Y. Wu, Number of spanning trees on a lattice, J. Phys. A 10, L113L115 (1977).

[23] R. Shrock and F. Y. Wu, Spanning trees on graphs and lattices in $d$ dimensions, J. Phys. A 33, 3881-3902 (2000).

[24] R. J. Baxter, F model on a triangular lattice, J. Math. Phys. 10, 12111216 (1969).

[25] F. Y. Wu, unpublished.

[26] A. J. Phares and F. J. Wunderlich, Thermodynamics and molecular freedom of dimers on plane honeycomb and kagomé lattices, Il Nuovo Cimento 301 B, 653-685 (1988). 
[27] V. Elser, Nuclear antiferromagnetism in a registered ${ }^{3} \mathrm{He}$ solid, Phys. Rev. Lett. 62, 2405-2408 (1989).

[28] F. Y. Wu, Dimer enumeration as a vertex model problem, preprint.

[29] C. S. Hsue, K. Y. Lin, and F. Y. Wu, Staggered 8-vertex model, Phys. Rev. B 12, 429-437 (1975).

[30] M. E. Fisher, On the dimer solution of planar Ising models, J. Math. Phys. 7, 1776-1781 (1966).

[31] G. R. Allen, Dimer models for the antiferroelctric transition in copper formate tetrahydrate, J. Chem. Phys. 60, 3299-3309 (1974).

[32] S. R. Salinas and J. F. Nagle, Theory of the phase transition in the layered hydrogen-bonded $\mathrm{SnCl}^{2} \cdot 2 \mathrm{H}_{2} \mathrm{O}$ crystal, Phys. Rev. B 9, 49204931 (1974).

[33] J. F. Nagle, C. S. O. Yokoi, and S. M. Bhattacharjee, Dimer models on anisotropic lattices, in Phase Transitions and Critical Phenomena, Vol 13, Eds. C. Domb and J. L. Lebowitz (Academic Press, New York 1989). 
Figure Captions

Fig. 1. Edge orientation and unit cells of the checkerboard lattice. Shaded squares are repeated. Weights $z_{2}$ and $z_{4}$ are replaced by $i z_{2}$ and $i z_{4}$ inn the evaluation of the Pfaffian (see text).

Fig. 2. Edge orientation and unit cells of the triangular lattice.

Fig. 3. The kagomé lattice.

Fig. 4. Edge orientation and a unit cell of the 3-12 lattice.

Fig. 5. Edge orientation and a unit cell of the 4-8 lattice.

Fig. 6. (a) The $\left[4 \cdot 8^{2}\right]$ Union Jack lattice, the dual of the 4-8 lattice. (b) The $\left[3 \cdot 12^{2}\right]$ lattice, the dual of the 3-12 lattice. 


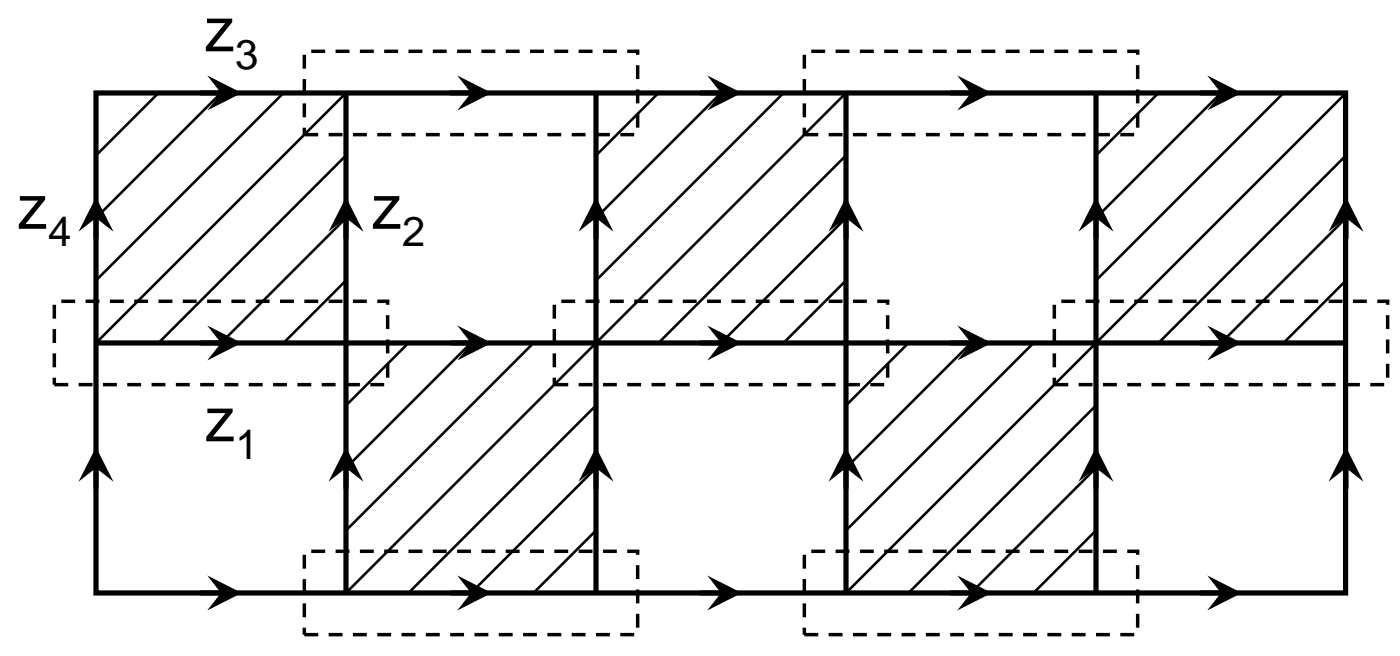

Fig. 1 


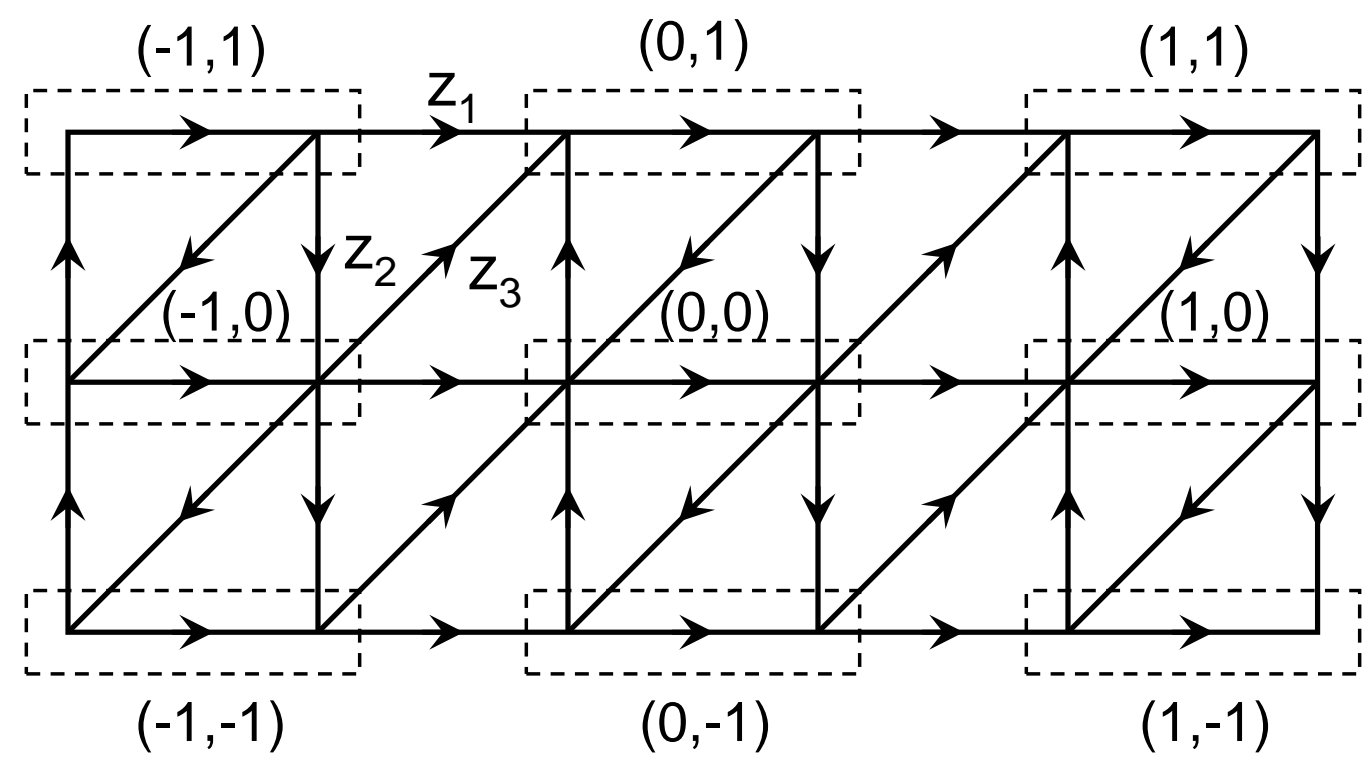

Fig. 2 


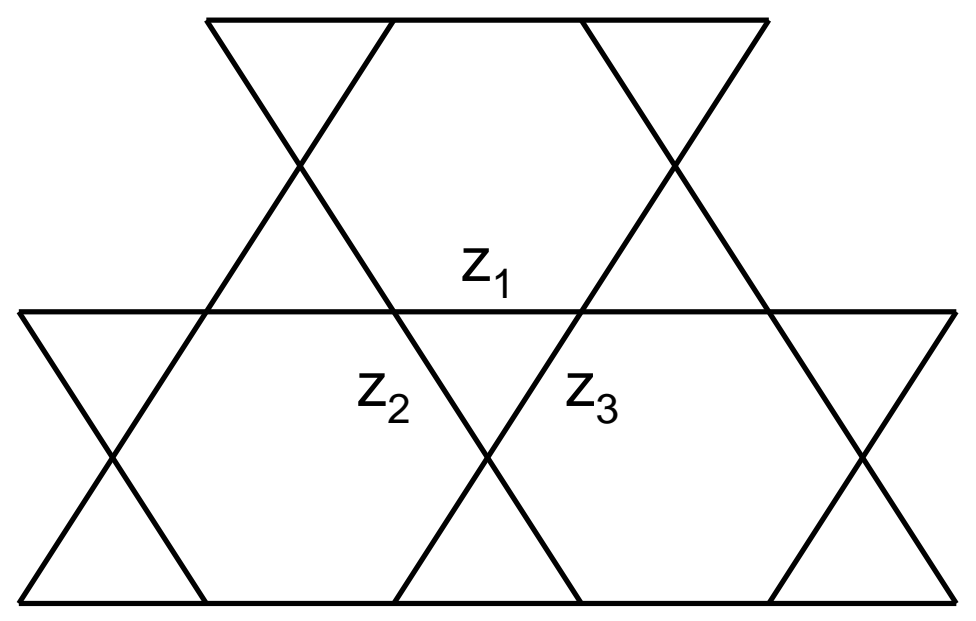

Fig. 3 


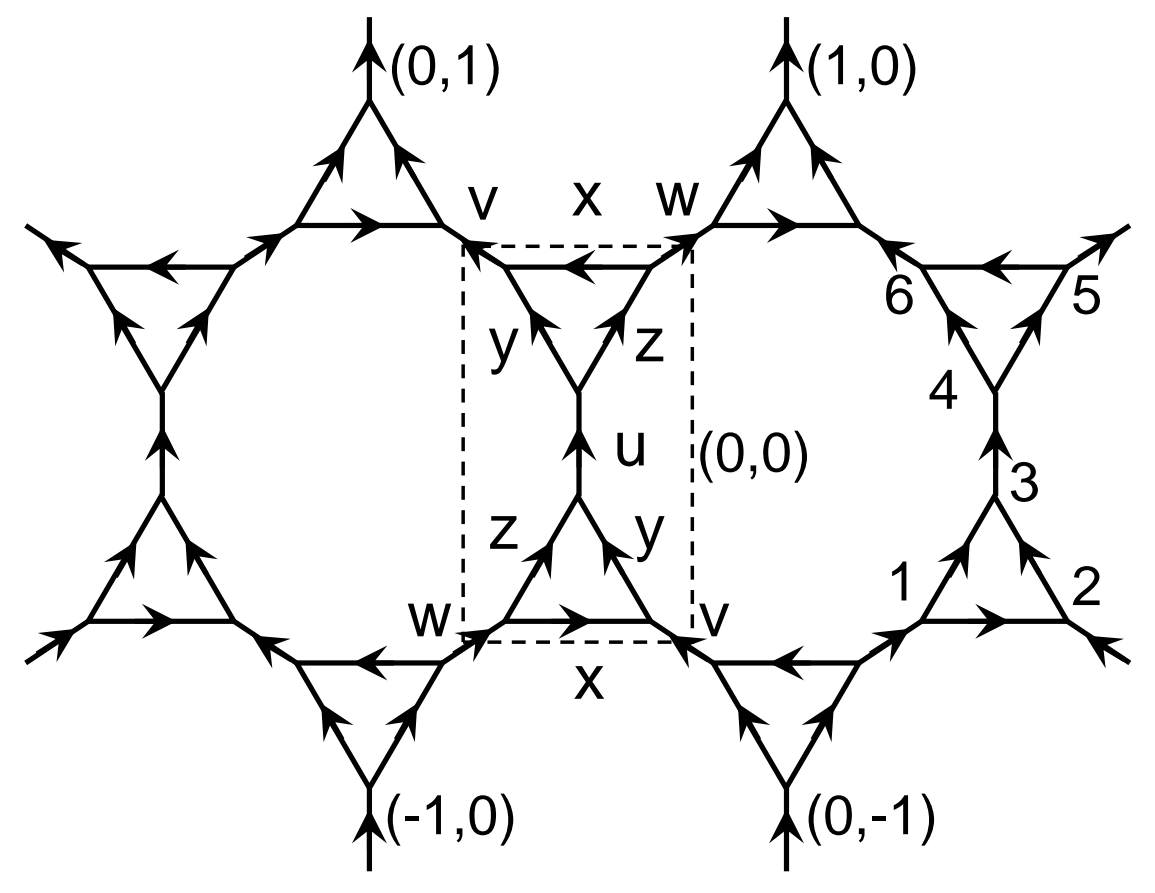

Fig. 4 


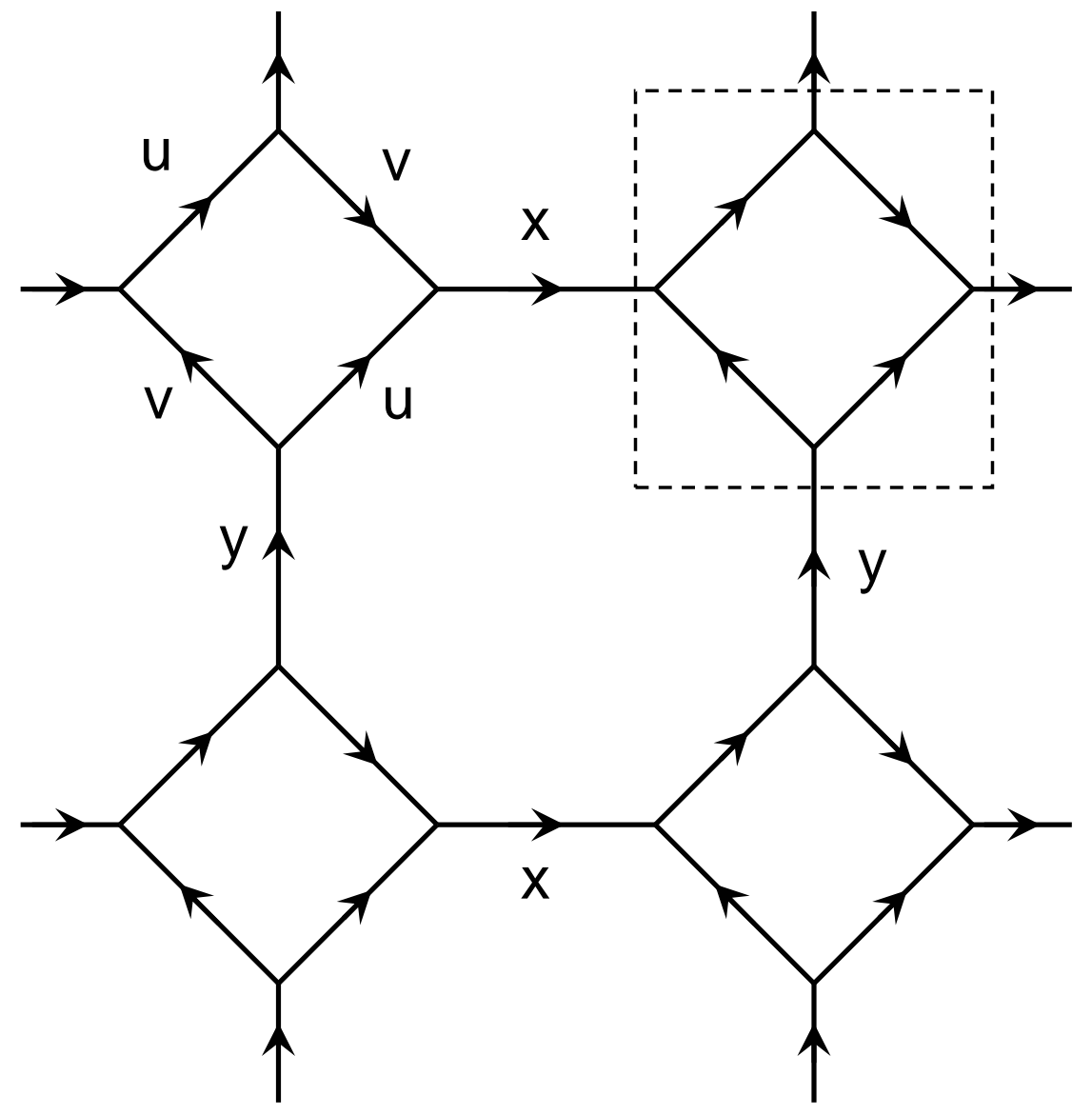

Fig. 5 


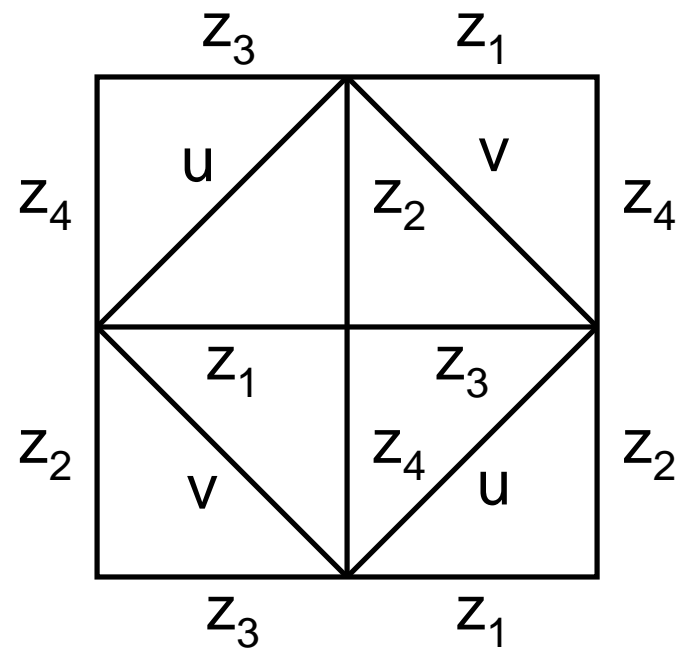

(a)

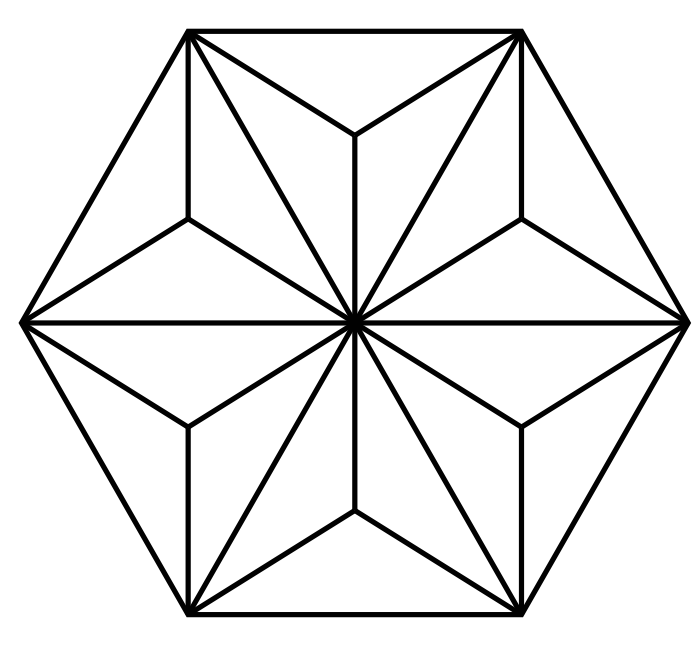

(b)

Fig. 6 